\title{
Radical First Contact: Bridging Astrobiology and SETI
}

\author{
Michael P. Oman-Reagan ${ }^{1}$ \\ Vanier Scholar (Social Sciences and Humanities Research Council of Canada) \\ Department of Anthropology, Memorial University, St. John's, NL A1C 557, Canada \\ Affiliated Researcher, University of Victoria, Victoria, BC V8P 5C2, Canada
}

\begin{abstract}
The astrobiological search for life in the universe is rarely discussed in terms of communicative first contact, a concept often reserved for the search for extraterrestrial intelligence (SETI). What are the implications for astrobiology of inheriting historical conclusions that there is no value in attempting communication with seemingly non-intelligent life on Earth? By imagining a radical first contact protocol, to bridge work in astrobiology and SETI, this paper challenges the assumption that certain forms of life elsewhere (those we might categorize as like microbial, plant, insect, computer, geologic, or other non-human forms and systems) can be eliminated in advance from attempts at communicative or cultural first contact. A radical first contact protocol asks that we push the limits of both our astrobiological and anthropological imaginations beyond the traditional scope of anthropological subjects and functional SETI-focused definitions of intelligence. By drawing on anthropological theory, ontological anthropology, multispecies ethnography, decolonizing methodologies, speculative fiction, as well as from fieldwork with astrobiologists and SETI scientists (Wright and Oman-Reagan 2017), this paper proposes how and why a radical first contact protocol might approach any potential life, broadly defined, as though it is also a potential intelligence, culture, or agent inviting communicative contact and moral consideration. By drawing on expansions of contemporary anthropology along with other theory and practice this project also aims to help scientists "step out of our brains" (Cabrol 2016) to build methods and insights which successfully bridge key aspects of first contact and the search for life in astrobiology and SETI.
\end{abstract}

Keywords: first contact, ontology, multispecies ethnography, decolonizing, astrobiology, SETI, anthropology

\section{Acknowledgements}

This research was supported by the Social Sciences and Humanities Research Council of Canada.

\section{References}

Cabrol, Nathalie. A. (2016). Alien Mindscapes-A Perspective on the Search for Extraterrestrial Intelligence. Astrobiology, 16(9), 661-676. https://doi.org/10.1089/ast.2016.1536

Wright, Jason T. and Michael P. Oman-Reagan (2017). Visions of Human Futures in Space and SETI. International Journal of Astrobiology. (in press)

\footnotetext{
1 omanreagan@mun.ca
} 